\title{
A spatial interpolation method based on radial basis function networks incorporating a semivariogram model
}

\author{
Gwo-Fong Lin*, Lu-Hsien Chen \\ Department of Civil Engineering, National Taiwan University, Taipei 10617, Taiwan \\ Received 8 May 2003; revised 30 September 2003; accepted 29 October 2003
}

\begin{abstract}
Based on the combination of the radial basis function network (RBFN) and the semivariogram, a spatial interpolation method, named improved RBFN, is proposed in this paper. To evaluate the interpolation accuracy of the proposed method, reference surfaces with prescribed semivariograms of different sills and scale parameters are generated. The proposed method as well as two existing methods (ordinary kriging and standard RBFN) is then used in the restoration of these reference surfaces. Among three interpolation methods, the proposed method has the highest interpolation accuracy regardless of the arrangement of sample points. The proposed method is performing well especially when the variance of the reference surface is large. An application of the proposed method to the estimation of the spatial distribution of rainfall also shows that the proposed method can estimate more precisely as compare to the other two existing methods. The proposed method is recommended as an alternative to the existing methods, because it has a clear principle and a simple structure. In addition, it provides more flexibility adjusted with stochastic property.
\end{abstract}

(C) 2003 Elsevier B.V. All rights reserved.

Keywords: Spatial interpolation; Radial basis functions; Kriging; Semivariogram

\section{Introduction}

Spatial interpolation of population characteristic values from data that are limited in number and obtained at irregularly arranged points is an important process for further understanding geostatistical structure in the natural fields. Geostatistics provides a set of statistical tools for analyzing spatial variability and spatial interpolation. A semivariogram is used to describe the structure of spatial variability. Kriging provides a best linear unbiased estimation for spatial

\footnotetext{
* Corresponding author. Fax: + 886-2-23631558

E-mail address: gflin@ntu.edu.tw (G.-F. Lin).
}

interpolation. Nowadays, geostatistics has become a popular means to describe spatial patterns and to interpolate the attribute of interest at unsampled locations. The geostatistical methods are increasingly used in many disciplines, such as mining, meteorology, hydrology, geology, remote sensing, soil science, ecology, and environmental science (Chirlin and Dagan, 1980; Bastin et al., 1984; Hill and Alexandar, 1989; White et al., 1997; Duc et al., 2000).

In the process of kriging analysis, the semivariogram plays a central role in the analysis of geostatistical data. A valid semivariogram model is selected and the model parameters are estimated before kriging is performed. These inference 
procedures are generally based upon examination of the experimental semivariogram, which consists of average squared differences of data taken at sites lagged the same distance. The calculation of the experimental semivariogram has a great influence on the accuracy of the model. On the other hand, if the experimental semivariogram is not well fitted, the kriging will not yield the best interpolation. Moreover, kriging has a disadvantage that it can be rather slow for larger data sets.

Neural networks, which devise via imitating brain activity and are capable of modeling nonlinear and complex systems, provide an alternative approach for hydrosystem modeling. Artificial neural networks were first developed in the 1940s. Generally speaking, neural networks are information processing systems. In recent decades, considerable interest has been raised over their practical applications, because the current algorithms can overcome the limitations of early networks. Bypassing the model construction and parameter estimation phases adopted by most of the conventional techniques, the neural networks can automatically develop a forecasting model through a simple process of the historic data. Such a training process enables the neural system to capture the complex and nonlinear relationships that are not easily analyzed using conventional methods. Based on the structure of the neural networks and the learning algorithm, various neural network models are frequently proposed and studied to solve different problems. The back-propagation network is the popular one. However, the back-propagation network has some disadvantages. For example, it tends to yield local solutions, the learning rate is slow and the network structure is difficult to develop. The architecture and training algorithms for radial basis function networks (RBFNs) are simple and clear. The RBFNs train more quickly than multiple layered perceptron networks. Unlike multiple layered perceptron networks, RBFNs allow for explanation when interpreted as fuzzy rule-based systems (Looney, 2002).

Radial basis functions were proposed in 1964 as potential functions (Aizerman et al., 1964; Bashkirov et al., 1964). RBFNs were first introduced to solve the real multivariate interpolation problem (Powell, 1987). Broomhead and Lowe (1988) were the first ones to exploit the use of radial basis functions in the design of neural networks. RBFNs have been employed in nonlinear systems identification and time series prediction (Moody and Darken, 1989). Poggio and Girosi (1990) developed regularization networks from approximation theory with RBFNs as a special case. Park and Sandberg (1991) studied the universal approximation problem using the RBFNs. More recently, RBFNs have been used in a wide variety of applications, such as radar target recognition (Zhao and Bao, 1996), curve fitting (Xu, 1998), hardware integration of neurocomputers in an avionics system (Sanchez et al., 1998), classification of mammograms (Tsujii et al., 1999).

However, for the learning of RBFNs, the spatial correlation has been neglected in the past. Such a limitation has prompted a search for an improved approach to estimating the most suitable parameters. In this paper, a spatial interpolation method is developed. The method is based on the RBFN incorporating a semivariogram model. Restoration problems of known reference surfaces from randomly selected sample data are used to examine the interpolation accuracy of the proposed method. Then the method is applied to an actual watershed to estimate the spatial distribution of rainfall.

\section{The proposed spatial interpolation method}

In this section, the structure of RBFN is introduced. Furthermore, a spatial interpolation method, which is based on the RBFN incorporating a semivariogram model, is developed. The proposed interpolation method is referred to as Improved RBFN (IRBFN) method herein.

\subsection{Standard RBFN}

RBFN can be presented as a three-layer feedforward structure. The basic architecture of a three-layered neural network is shown in Fig. 1. The input data $X$ is an $L$-dimensional vector, $X=\left[x_{1}, x_{2}, \ldots, x_{L}\right]^{\mathrm{T}}$. The input layer serves only as input distributor to the hidden layer. The dimensionality of hidden units is the same as that of the input data. The response from the $j$ th hidden unit for the $i$ th input data $x_{i}$ has the following form

$\phi_{j}\left(x_{i}\right)=\phi\left(\left\|x_{i}-c_{j}\right\|\right) \quad j=1,2, \ldots, M$ 


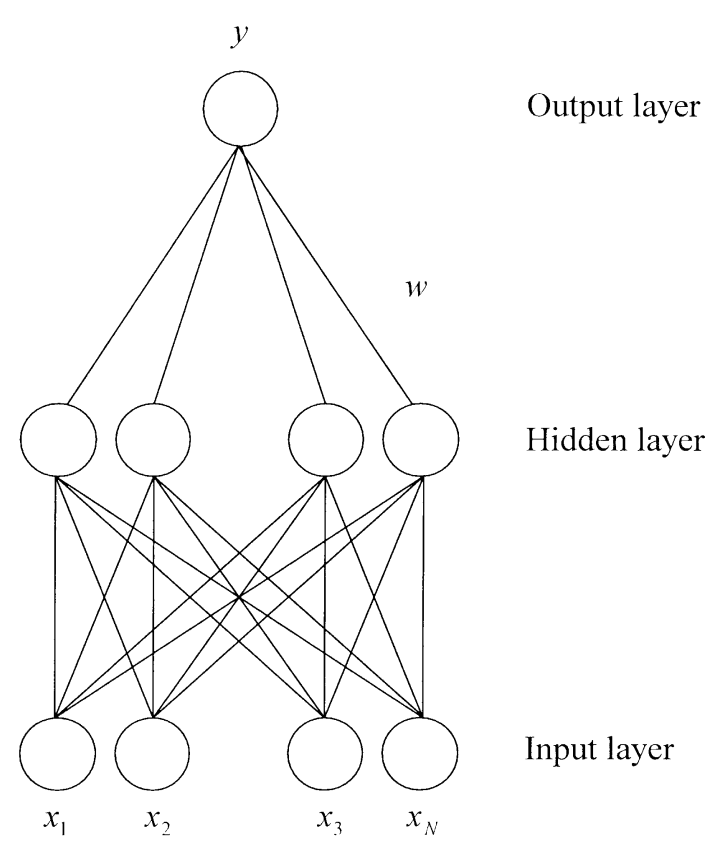

Fig. 1. The structure of RBFN.

where \|\| denotes the Euclidean norm, $c_{j}$ is the center of the $j$ th unit (neuron) in the hidden layer, $\phi()$ is the activation function, and $M$ is the number of hidden units. In the spatial interpolation, $c_{j}$ represents the $j$ th observed data point, and $\left\|x_{i}-c_{j}\right\|$ denotes the distance between the $j$ th observed data point and the $i$ th estimated location. The activation function is a nonlinear function that is radially symmetric in the input space. The response of the $j$ th hidden unit depends only on the radial distance between the center of the jth hidden unit and the input vector. The activation function can have different forms, for example, the Gaussian function

$\phi(X)=\exp \left(-\frac{\left\|X-c_{j}\right\|^{2}}{2 \sigma^{2}}\right)$

where $\sigma$ is the width of the hidden units and $\sigma$ can be obtained from (Haykin, 1994)

$\sigma=\frac{d_{\max }}{\sqrt{2 M}}$

where $d_{\max }$ is the maximum distance between the centers of hidden units and $M$ is the number of hidden units.
For an output layer of $N$ units, the output of the $k$ th unit, $\hat{y}_{k}$, can be obtained from

$\hat{y}_{k}=\sum_{j=1}^{M} w_{j k} \phi_{j}(x) \quad k=1,2, \ldots, N$

where $\phi_{j}(x)$ is the response of the $j$ th hidden unit resulting from all input data, $w_{j k}$ is the connecting weight between the $j$ th hidden unit and the $k$ th output unit, and $N$ is the number of output units.

In matrix notation, Eq. (4) becomes

$Y=W \Phi$

where

$W=\left[\begin{array}{cccc}w_{11} & w_{21} & \cdots & w_{M 1} \\ w_{12} & w_{22} & \cdots & w_{M 2} \\ \cdot & \cdot & \cdot & \cdot \\ w_{1 N} & w_{2 N} & \cdots & w_{M N}\end{array}\right]$

and

$\Phi=\left[\phi_{1} \phi_{2} \cdots \phi_{M}\right]^{\mathrm{T}}$

\subsection{Improved $R B F N$}

In RBFNs, each hidden unit has two parameters: center $c$ and width $\sigma$. It is often assumed that the number of hidden units is significantly less than the number of data points. However, for the space interpolation method, the number of hidden units (neurons) equals the number of known data. Therefore, the calculation of width $\sigma$ in each hidden unit becomes an important problem for the construction of an RBFN. The width $\sigma$ controls the smoothness property of the activation function. When the width $\sigma$ is small, the corresponding area of the representation space is small. Hence, a high number of centers will be required during the process of training. This results in over-parameterization. On the contrary, the area of the representation space may be too extensive when the width $\sigma$ is large. For both cases, the generalization capabilities of the network will be poor (Oukhellou and Aknin, 1999).

Since Eq. (3) is deterministic, the activation function of Eq. (2) does not have any stochastic property. However, the spatial variables correlate to each other within a range. Covariation analysis 
displays the data in ways that reveal the correlation structure of the spatial data. The experimental semivariogram is the most important tool for covariation analysis. The experimental semivariogram represents the spatial variability in the data and is employed to determine the optimal weights used with kriging methods. Based on the combination of the RBFN and the semivariogram of kriging, a spatial interpolation method, named IRBFN, is proposed below.

The exponential form of the activation function for the hidden layer is adopted herein for developing the proposed spatial interpolation method. The exponential activation function is defined as

$\phi_{j}(x)=\exp \left(-\frac{h}{\sigma_{j}}\right)$

where $h=\left\|x-c_{j}\right\|$ and $\sigma_{j}$ is the width of the $j$ th hidden unit. It should be noted that the hidden units have different widths for the exponential activation function. On the contrary, all hidden units have the same width for the Gaussian activation function of Eq. (2).

The exponential form of the covariance function of semivariogram is popular in hydrologic applications (Kitanidis, 1993)

$C(h)=C(0) \exp \left(-\frac{h}{a}\right)$

where $C(0)$ is the variance, $a$ is the scale parameter, and $C(h)$ is the covariance for distance $h$. Eq. (9) can be further written as

$\frac{C(h)}{C(0)}=\exp \left(-\frac{h}{a}\right)$

Eqs. (8) and (10) have the same form. Hence, once the covariance function is known, the width $\sigma_{j}$ can be found. It should be noted that $\sigma_{j}$ obtained in this manner is the same for all hidden units because there is only one covariance value. To obtain the covariance function, one has to know the mean first. In practice, the mean is estimated from the observed data, which introduces a bias. Thus, the sample covariance also has a bias and results in inaccurate spatial interpolation. To avoid such a problem, the semivariogram will be used to estimate the width $\sigma_{j}$.
The semivariogram can automatically filter the mean (Chiles and Delfiner, 1999).

The semivariogram $\gamma(h)$ is related to the covariance function $C(h)$ through

$\gamma(h)=C(0)-C(h)$

Inserting Eqs. (9) into Eq. (11) gives the exponential semivariogram model

$\gamma(h)=C(0)\left[1-\exp \left(-\frac{h}{a}\right)\right]$

where the variance $C(0)$ is referred to as the sill. It should be noted that the range is $\alpha \approx 3 a$ (Kitanidis, 1993).

Eqs. (8) and (12) have the same form. In a like manner, one can obtain the width $\sigma_{j}$ in Eq. (8), once the experimental semivariogram is known. This still leads to a constant $\sigma_{j}$ for all hidden units because there is only one semivariogram available. On the other hand, the experimental semivariogram is calculated from all sample points, and hence it cannot present the variety of small regions. An algorithm, that can account for the variety of small regions and yield different values of $\sigma_{j}$ for different hidden units, is proposed below.

For each hidden unit one can let the range $\alpha$ as a neighborhood, and then compute the average distance $\bar{d}_{n}$ of the sample points in this neighborhood. Half the average distance $\left(\bar{d}_{n} / 2\right)$ in the $j$ th hidden unit's neighborhood represents the width of the $j$ th hidden unit. The use of $\bar{d}_{n} / 2$ is determined by experimentation herein. Among ratios of the average distance such as $\bar{d}_{n} / 2, \bar{d}_{n} / 3, \bar{d}_{n} / 4$, etc. half the average distance has the best interpolation accuracy. Two extreme cases of neighborhood are treated as follows. First, if the neighborhood is smaller than half the minimum distance between the $j$ th hidden unit and its nearest sample point (i.e. there is no sample point within this neighborhood), the value of $\sigma_{j}$ is set equal to half Euclidean distance between the $j$ th hidden unit and its nearest sample point. Second, if the neighborhood is larger than half the maximum distance between all sample points, the width of the jth hidden unit is set equal to half the average distance between all sample points. 


\section{Evaluation of interpolation accuracy}

To evaluate the interpolation accuracy of the proposed method, three cases of semivariograms are considered and their corresponding random fields are generated. The random fields are obtained by HYDRO_GEN (Bellin and Rubin, 1996). The HYDRO_GEN can produce rapidly and accurately random fields of very large dimensions. One can refer to the work of Bellin and Rubin (1996) for the advantages of HYDRO_GEN over the turning bands method and the fast Fourier transform based method.

Each random field is a square area that is divided equally into 81 squares to give 100 grid points. Each grid is 1 by 1 unit. Then, 25 points are randomly selected from the random field. These 25 points are then used to estimate the other 75 points using an interpolation method. The difference of the value at the same point between the original and the estimated ones is used to evaluate the interpolation accuracy.
It should be noted the generated random field is also referred to as the reference surface herein. In the following three cases, the proposed spatial interpolation method will be used in the restoration of the surfaces.

\subsection{Isotropic semivariogram with sill $C(0)=1$ and scale parameter $a=1$}

Fig. 2 presents the isotropic reference surface (random field) produced from Eq. (12) with sill $C(0)=1$ and scale parameter $a=1$. Fig. 3 presents the fitted and experimental semivariograms based on the sample data in Fig. 2. The fitted semivariogram almost agrees with the $\gamma(h)$ defined in Eq. (12) with sill $C(0)=1$ and scale parameter $a=1$.

Then, 10 different arrangements of the 25 grid points are prepared by selected randomly from the 100 data in Fig. 2. For each arrangement, the root mean square error (RMSE) is used to evaluate

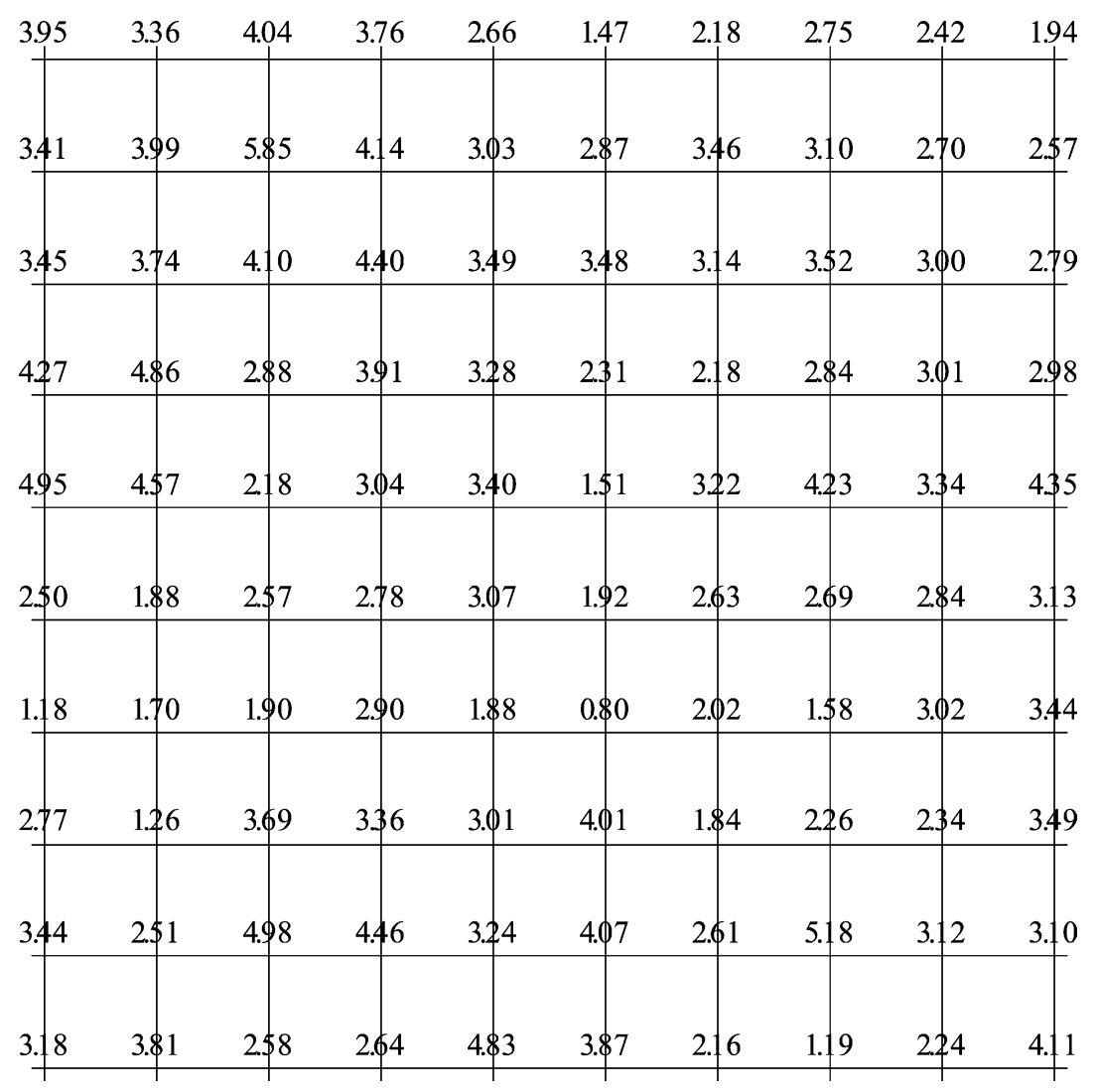

Fig. 2. Isotropic reference surface with $C(0)=1$ and $a=1$. 


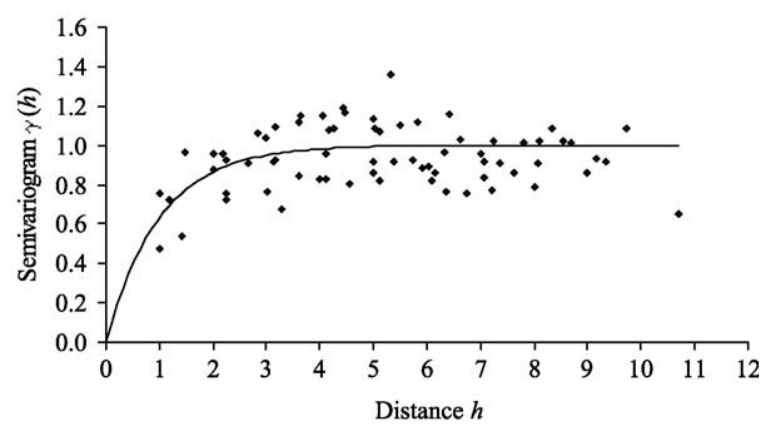

Fig. 3. The fitted and experimental semivariograms based on the sample data in Fig. 2.

the interpolation accuracy

$\mathrm{RMSE}=\sqrt{\frac{1}{N_{\mathrm{e}}} \sum_{p=1}^{N_{\mathrm{e}}}\left[\frac{\hat{Z}_{p}-Z_{p}}{Z_{p}}\right]^{2}}$

where $N_{\mathrm{e}}$ is the number of estimated values, $\hat{Z}_{p}$ is the estimated value at the $p$ th grid point, and $Z_{p}$ is the value of the reference surface at the $p$ th grid point. The RMSE can be used to compare the performance of several interpolation methods. The method that yields the smallest value of RMSE is the best.

The RMSE values for the interpolation results using IRBFN are summarized in Table 1 . These results are compared with those using the Ordinary Kriging (OK) and the Standard RBFN (SRBFN). It should be noted that the SRBFN uses Eq. (2) as the activation

Table 1

Comparison of interpolation accuracy of the three methods, IRBFN, OK and SRBFN, based on RMSE for 10 arrangement patterns of sample points selected randomly from Fig. 2

\begin{tabular}{llll}
\hline Pattern & \multicolumn{2}{l}{ RMSE } & \\
\cline { 2 - 4 } & IRBFN & OK & SRBFN \\
\hline 1 & 0.375 & 0.442 & 0.405 \\
2 & 0.423 & 0.627 & 0.552 \\
3 & 0.447 & 0.479 & 0.485 \\
4 & 0.411 & 0.485 & 0.663 \\
5 & 0.451 & 0.543 & 0.628 \\
6 & 0.414 & 0.483 & 0.488 \\
7 & 0.457 & 0.562 & 0.482 \\
8 & 0.427 & 0.520 & 0.716 \\
9 & 0.418 & 0.615 & 0.483 \\
10 & 0.511 & 0.612 & 0.549 \\
Mean & 0.433 & 0.537 & 0.545 \\
\hline
\end{tabular}

function and employs only Eq. (3) to estimate the width of the hidden units. IRBFN achieves RMSE values ranging from 0.375 to 0.511 and has the lowest RMSE values. IRBFN yields an average RMSE reduction of $19 \%$ as compared to OK, and $20 \%$ as compared to SRBFN. IRBFN has the smallest RMSE values for all arrangement patterns and the variation of the RMSE values is small. The validity and stability of IRBFN is clearly demonstrated.

\subsection{Isotropic semivariogram with sill $C(0)=1$ and scale parameter $a=2$}

The isotropic reference surface generated with sill $C(0)=1$ and scale parameter $a=2$ in Eq. (12) is shown in Fig. 4. The corresponding fitted and experimental semivariograms are presented in Fig. 5. As shown in Fig. 5, the fitted semivariogram is close to the prescribed semivariogram with sill $C(0)=1$ and scale parameter $a=2$.

In a like manner, 10 arrangement patterns consisting of 25 sample points are made. The comparison of interpolation accuracy of the three methods based on RMSE for these 10 arrangement patterns of sample points is summarized in Table 2 . Table 2 shows that IRBFN achieves RMSE values ranging from 0.292 to 0.418 . IRBFN yields an average RMSE reduction of $19 \%$ as compared to OK, and $26 \%$ as compared to SRBFN. It is noteworthy that the small interpolation error can be obtained by IRBFN for any arrangement pattern.

\subsection{Isotropic semivariogram with sill $C(0)=2$ and scale parameter $a=1$}

In a like manner, the reference surface is shown in Fig. 6 and the corresponding fitted and experimental semivariograms are presented in Fig. 7. As shown in Fig. 7, the fitted semivariogram is in good agreement with the prescribed semivariogram with sill $C(0)=2$ and scale parameter $a=1$. Table 3 gives the RMSE values for the interpolation results by three methods. The results in Table 3 are similar to those in Tables 1 and 2. As shown in Table 3, one can find that IRBFN achieves RMSE values ranging from 0.545 to 2.667. The reduction in average RMSE obtained by IRBFN compared to OK exceeds $30 \%$ and is typically $33 \%$. 


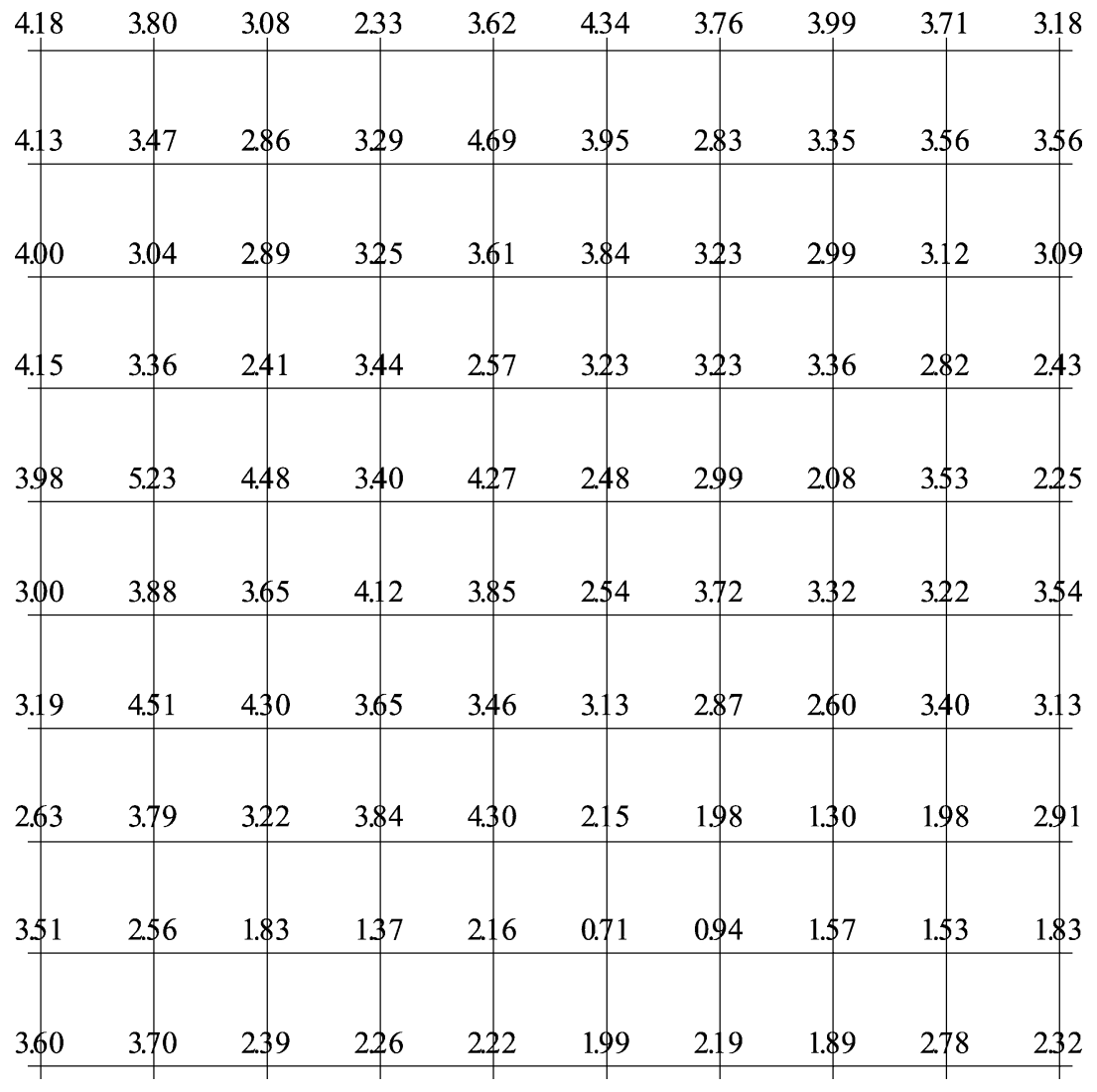

Fig. 4. Isotropic reference surface with $C(0)=1$ and $a=2$.

Similar reduction in average RMSE is obtained compared to SRBFN.

Based on the results of these three cases, the RMSE values obtained by IRBFN are smaller than those obtained by OK and SRBFN. Therefore, IRBFN can be regarded as an interpolation method with high

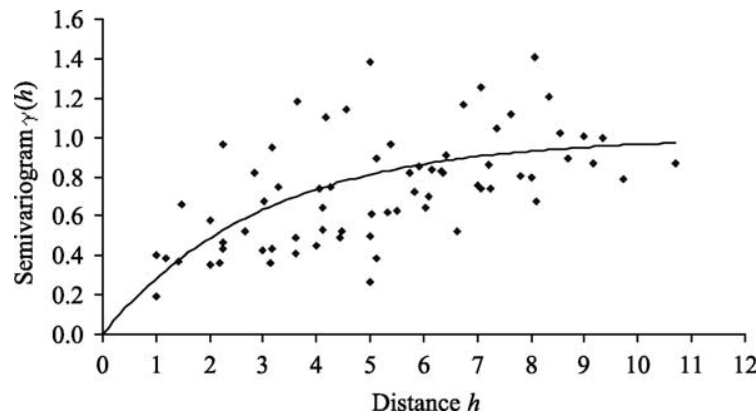

Fig. 5. The fitted and experimental semivariograms based on the sample data in Fig. 4.
Table 2

Comparison of interpolation accuracy of the three methods based on RMSE for 10 arrangement patterns of sample points selected randomly from Fig. 4

\begin{tabular}{llll}
\hline Pattern & \multicolumn{2}{l}{ RMSE } & \\
\cline { 2 - 4 } & IRBFN & OK & SRBFN \\
\hline 1 & 0.363 & 0.381 & 0.411 \\
2 & 0.330 & 0.348 & 0.456 \\
3 & 0.329 & 0.381 & 0.344 \\
4 & 0.327 & 0.420 & 0.487 \\
5 & 0.418 & 0.602 & 0.446 \\
6 & 0.367 & 0.385 & 0.440 \\
7 & 0.326 & 0.469 & 0.391 \\
8 & 0.353 & 0.422 & 0.490 \\
9 & 0.407 & 0.619 & 0.835 \\
10 & 0.292 & 0.308 & 0.432 \\
Mean & 0.351 & 0.433 & 0.473 \\
\hline
\end{tabular}




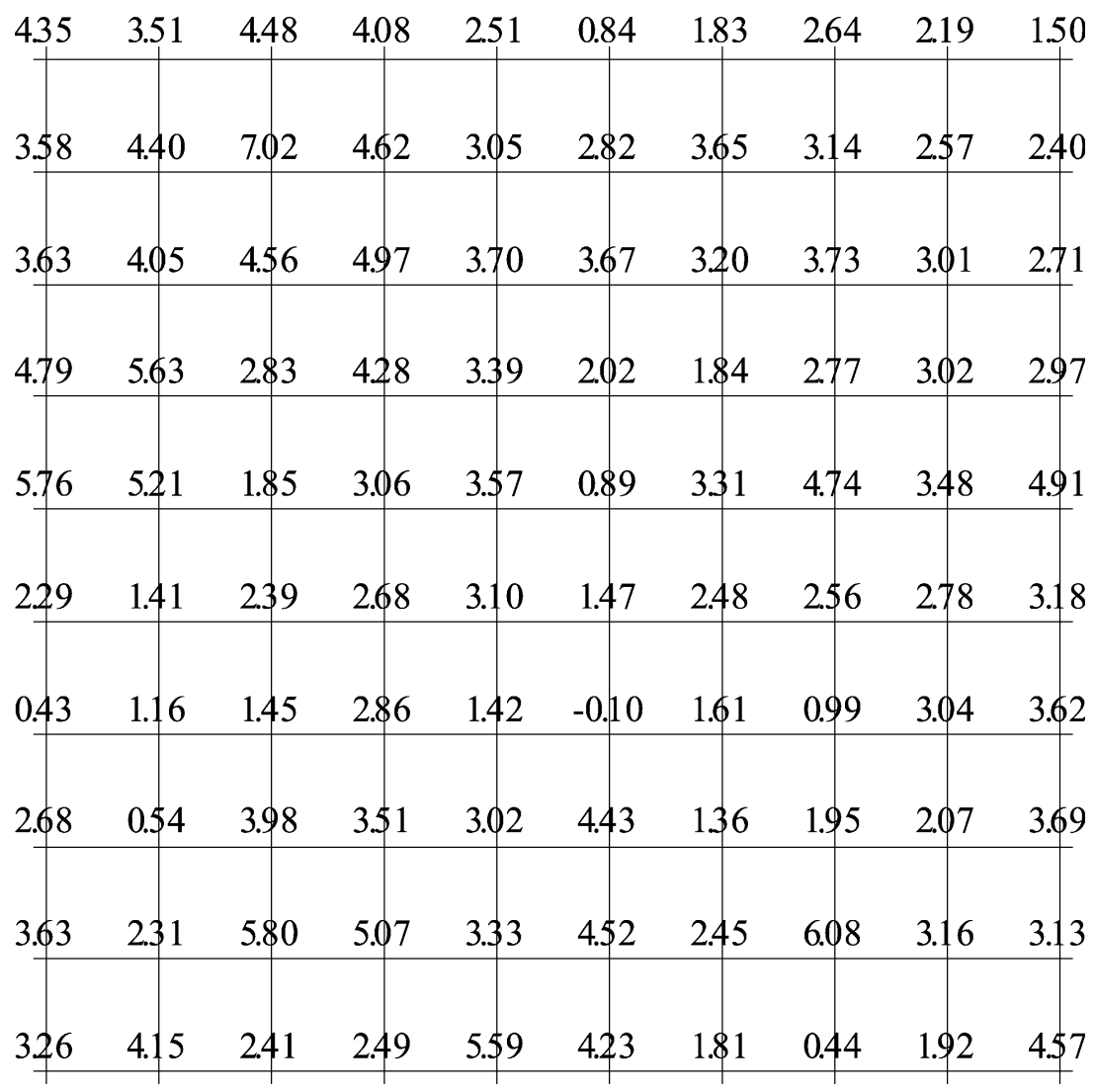

Fig. 6. Isotropic reference surface with $C(0)=2$ and $a=1$.

accuracy. In addition, one can find that the variance has a great influence on evaluating the interpolation accuracy. Especially when the variance of the reference surface is large, the range of reduction in average RMSE obtained by IRBFN becomes greater. In other words, IRBFN is performing well when the variance of the reference surface is large.

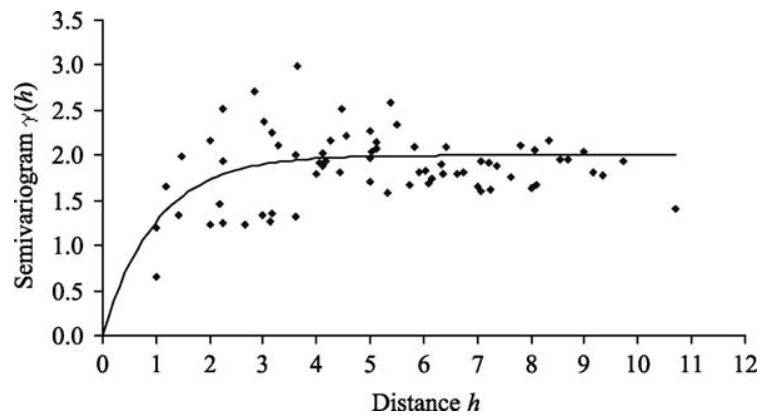

Fig. 7. The fitted and experimental semivariograms based on the sample data in Fig. 6.
Table 3

Comparison of interpolation accuracy of the three methods based on RMSE for 10 arrangement patterns of sample points selected randomly from Fig. 6

\begin{tabular}{llll}
\hline Pattern & \multicolumn{2}{l}{ RMSE } & \\
\cline { 2 - 4 } & IRBFN & OK & SRBFN \\
\hline 1 & 0.898 & 1.150 & 1.356 \\
2 & 2.667 & 3.024 & 3.655 \\
3 & 0.545 & 1.001 & 0.741 \\
4 & 0.837 & 1.136 & 1.193 \\
5 & 0.697 & 1.226 & 1.329 \\
6 & 0.553 & 0.950 & 1.240 \\
7 & 0.594 & 0.949 & 1.256 \\
8 & 1.734 & 2.734 & 2.088 \\
9 & 1.584 & 2.858 & 1.814 \\
10 & 1.576 & 2.393 & 1.911 \\
Mean & 1.168 & 1.742 & 1.658 \\
\hline
\end{tabular}




\section{Application}

The proposed spatial interpolation method is applied to estimate the spatial distribution of rainfall in the Tanshui River Basin in northern Taiwan. The average slope of the Tanshui River is $1 / 45$, and the area of the basin is $2726 \mathrm{~km}^{2}$. There are 55 rain gauges in this basin. The rainfall data are collected from computer archives of the Central Weather Bureau. Fig. 8 shows the study area and the locations of 55 rain gauges. In total, 64 sets of hourly rainfall records are used to compare the performance of the proposed method and the other two methods.

Cross-validation (leave-one-out-method) is selected to check the performance of the three models herein. The principle of cross-validation is to estimate the value at each sample point from the other data. Thus at every sample point one can get an estimate. The true value being known, one can compute the RMSE. The interpolation method that yields the smallest value of error is the best method. Table 4 summarizes the RMSE values for the interpolation results by three methods. The average RMSE values for IRBFN, OK and SRBFN are $0.525,0.746$ and 0.793 , respectively. IRBFN yields an average RMSE reduction of $30 \%$ as compared to
OK, and $34 \%$ as compared to SRBFN. Globally, the best method is IRBFN although for some data sets OK and SRBFN perform as well or even better than IRBFN. The results demonstrate the accuracy and reliability of the interpolation method proposed in this paper.

\section{Summary and conclusions}

A spatial interpolation method based on RBFNs incorporating a semivariogram model has been developed. The method is named Improved RBFN (IRBFN). The IRBFN, with a clear principle and a simple structure, provides more flexibility adjusted with stochastic property. Moreover, IRBFN has the higher computation speed for larger data set as compared to kriging. To evaluate the interpolation accuracy, three reference surfaces, which are based on isotropic semivariograms with different sills and scale parameters, are defined. Among three interpolation methods (IRBFN, OK and SRBFN) used in the restoration of the surfaces, IRBFN is shown to have the highest interpolation accuracy regardless of the arrangement of the sample points. Actual application also shows that IRBFN can
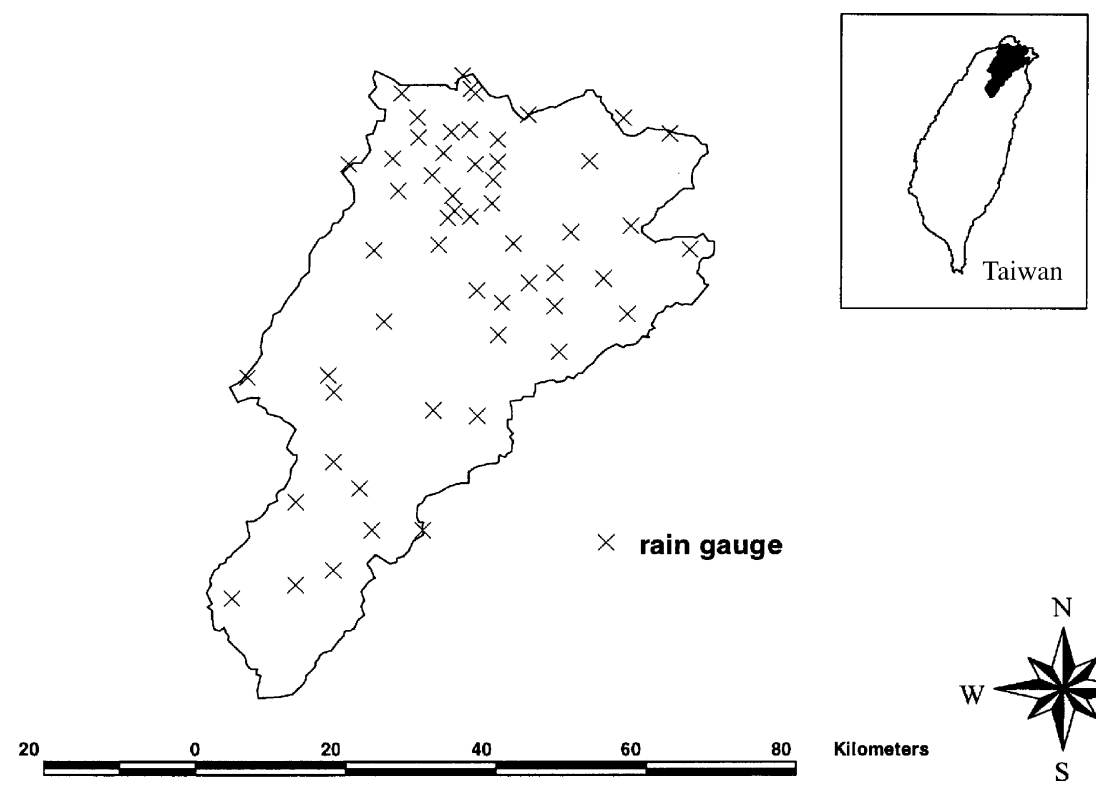

Fig. 8. Locations of rain gauges in the Tanshui River Basin in northern Taiwan. 
Table 4

Comparison of interpolation accuracy of the three methods based on RMSE for 64 data sets

\begin{tabular}{|c|c|c|c|c|c|c|c|}
\hline \multirow[t]{2}{*}{ Data set } & \multicolumn{3}{|l|}{ RMSE } & \multirow[t]{2}{*}{ Data set } & \multicolumn{3}{|l|}{ RMSE } \\
\hline & IRBFN & $\mathrm{OK}$ & SRBFN & & IRBFN & OK & SRBFN \\
\hline 1 & 0.535 & 0.591 & 0.650 & 33 & 0.495 & 0.899 & 0.602 \\
\hline 2 & 0.485 & 0.506 & 0.894 & 34 & 0.345 & 0.522 & 0.653 \\
\hline 3 & 0.437 & 0.555 & 0.682 & 35 & 0.723 & 0.807 & 1.142 \\
\hline 4 & 0.504 & 0.758 & 0.699 & 36 & 0.815 & 0.935 & 0.835 \\
\hline 5 & 0.400 & 0.570 & 0.638 & 37 & 0.563 & 0.913 & 0.714 \\
\hline 6 & 0.948 & 0.957 & 1.350 & 38 & 0.522 & 0.688 & 0.691 \\
\hline 7 & 0.439 & 0.456 & 0.683 & 39 & 0.621 & 0.838 & 0.606 \\
\hline 8 & 0.438 & 0.412 & 0.588 & 40 & 0.366 & 0.660 & 0.688 \\
\hline 9 & 0.623 & 0.709 & 0.905 & 41 & 0.400 & 0.566 & 0.632 \\
\hline 10 & 0.937 & 1.140 & 1.459 & 42 & 0.383 & 0.606 & 0.535 \\
\hline 11 & 0.492 & 0.696 & 0.854 & 43 & 0.309 & 0.360 & 0.496 \\
\hline 12 & 0.636 & 0.844 & 0.948 & 44 & 0.425 & 0.474 & 0.605 \\
\hline 13 & 0.339 & 0.371 & 0.595 & 45 & 0.253 & 0.244 & 0.510 \\
\hline 14 & 0.469 & 0.808 & 0.838 & 46 & 0.275 & 0.283 & 0.512 \\
\hline 15 & 0.422 & 0.503 & 0.709 & 47 & 0.324 & 0.523 & 0.503 \\
\hline 16 & 0.489 & 0.877 & 0.804 & 48 & 0.355 & 0.358 & 0.503 \\
\hline 17 & 0.482 & 0.481 & 0.656 & 49 & 0.251 & 0.231 & 0.579 \\
\hline 18 & 0.678 & 1.175 & 0.848 & 50 & 0.299 & 0.426 & 0.495 \\
\hline 19 & 0.442 & 0.480 & 0.642 & 51 & 0.312 & 0.657 & 0.501 \\
\hline 20 & 0.447 & 0.474 & 0.607 & 52 & 0.404 & 0.469 & 1.009 \\
\hline 21 & 0.452 & 0.535 & 0.700 & 53 & 0.439 & 0.734 & 0.808 \\
\hline 22 & 0.537 & 0.618 & 0.545 & 54 & 0.631 & 0.979 & 0.830 \\
\hline 23 & 0.591 & 0.622 & 0.944 & 55 & 0.713 & 0.905 & 1.442 \\
\hline 24 & 0.766 & 1.738 & 1.670 & 56 & 0.637 & 0.737 & 1.303 \\
\hline 25 & 0.690 & 1.662 & 0.801 & 57 & 0.512 & 0.807 & 0.702 \\
\hline 26 & 0.733 & 1.144 & 1.033 & 58 & 0.716 & 1.159 & 0.878 \\
\hline 27 & 0.621 & 1.079 & 1.109 & 59 & 0.632 & 1.573 & 0.584 \\
\hline 28 & 0.712 & 1.941 & 1.220 & 60 & 0.410 & 0.494 & 0.680 \\
\hline 29 & 0.857 & 1.010 & 0.684 & 61 & 0.694 & 1.011 & 0.700 \\
\hline 30 & 0.474 & 0.799 & 0.929 & 62 & 0.810 & 0.883 & 1.645 \\
\hline 31 & 0.342 & 0.436 & 0.706 & 63 & 0.383 & 0.414 & 0.624 \\
\hline \multirow[t]{2}{*}{32} & 0.494 & 0.595 & 0.786 & 64 & 0.687 & 1.046 & 0.590 \\
\hline & & & & Mean & 0.525 & 0.746 & 0.793 \\
\hline
\end{tabular}

estimate the spatial distribution of rainfall more precisely as compared to the other two methods.

\section{References}

Aizerman, M.A., Braverman, E.M., Rozonoer, L.I., 1964 Theoretical foundations of the potential function method in pattern recognition learning. Automatic Remote Control 25, $821-837$.

Bashkirov, O.K., Braverman, E.M., Muchnik, I.B., 1964. Potential function algorithms for pattern recognition learning machines. Automatic Remote Control 25, 629-631.
Bastin, G., Lorent, B., Duque, C., Gevers, M., 1984. Optimal estimation of the average rainfall and optimal selection of raingage locations. Water Resources Research 20 (4), 463-470.

Bellin, A., Rubin, Y., 1996. HYDRO_GEN: a spatially distributed random field generator for correlated properties. Stochastic Hydrology and Hydraulics 10 (4), 253-278.

Broomhead, D.S., Lowe, D., 1988. Multilvariable functional interpolation and adaptive networks. Complex System 2, $321-355$.

Chiles, J.P., Delfiner, P., 1999. Geostatistics: Modeling Spatial Uncertainty. Wiley, New York.

Chirlin, G.R., Dagan, G., 1980. Theoretical head variogram for steady flow in statistically homogeneous aquifers. Water Resources Research 16 (6), 1001-1015. 
Duc, H., Shannon, I., Azzi, M., 2000. Spatial distribution characteristics of some air pollutants in Sydney. Mathematics and Computers in Simulation 54 (1-3), 1-21.

Haykin, S., 1994. Neural Networks: A Comprehensive Foundation. IEEE Press, New York.

Hill, M., Alexandar, F., 1989. Statistical methods used in assessing the risk of disease near a source of possible environmental pollution: a review. Journal of Royal Statistical Society 152, 353-363.

Kitanidis, P.K., 1993. Geostatistics. In: Maidment, D.R., (Ed.), Handbook of Hydrology, McGraw-Hill, New York, pp. 2.01-20.39.

Looney, C.G., 2002. Radial basis functional link nets and fuzzy reasoning. Neurocomputing 48, 489-509.

Moody, J., Darken, C.J., 1989. Fast learning in networks of locallytuned processing units. Neural Computation 1, 281-294.

Oukhellou, L., Aknin, P., 1999. Hybrid training of radial basis function networks in a partitioning context of classification. Neurocomputing 28, 165-175.

Park, J., Sandberg, I.W., 1991. Universal approximation using radial-basis-function networks. Neural Computation 3, $246-257$.
Poggio, T., Girosi, F., 1990. Networks for approximation and learning. Proceedings of the IEEE 78 (9), 1481-1497.

Powell, M.J.D., 1987. Radial basis functions for multivariable interpolation: a review. In: Mason, J.C., Cox, M.G. (Eds.), Algorithms for Approximation, Carendon Press, Oxford, pp. $143-167$.

Sanchez, V.D., Sloat, S., Guerrero, J., Shullo, D., Lefebvre, M., 1998. The design of a real-time neurocomputer based on RBF networks. Neurocomputing 20 (3), 111-114.

Tsujii, O., Freedman, M.T., Mun, S.K., 1999. Classification of microcalcifications in digital mammograms using trendoriented radial basis function neural network. Pattern Recognition 32 (5), 891-903.

White, J.G., Welch, R.M., Norvell, W.A., 1997. Soil zinc map of the USA using geostatistics and geographic information systems. Soil Science Society of America Journal 61 (1), 185-194.

$\mathrm{Xu}, \mathrm{L} ., 1998$. RBF nets, mixture experts, and Bayesian Ying-Yang learning. Neurocomputing 19 (3), 223-257.

Zhao, Q., Bao, Z., 1996. Radar target recognition using a radial basis function neural network. Neural Networks 9 (4), $709-720$. 